\title{
HTS Josephson junctions arrays for high-frequency mixing
}

A. Sharafiev ${ }^{1}$, M. Malnou ${ }^{1}$, C. Feuillet-Palma ${ }^{1}$, C. Ulysse $^{2}$, T. Wolf ${ }^{1}$, F. Couëdo ${ }^{1}$, P. Febvre ${ }^{3}$, J. Lesueur $^{1 *}$, N. Bergeal ${ }^{1}$

${ }^{1}$ Laboratoire de Physique et d'Etude des Matériaux, CNRS, ESPCI Paris, PSL Research University, UPMC, Paris, France.

${ }^{2}$ Centre de Nanosciences et de Nanotechnologie, CNRS, Université Paris Saclay, Marcoussis, France.

${ }^{3}$ IMEP-LAHC, CNRS, Université Savoie Mont Blanc, Le Bourget du Lac, France.

\begin{abstract}
We designed, fabricated and measured short one-dimensional arrays of masked ion-irradiated $\mathrm{YBa}_{2} \mathrm{Cu}_{3} \mathrm{O}_{7}$ Josephson junctions embedded into log-periodic spiral antennas. They consist of 4 or 8 junctions separated either by $960 \mathrm{~nm}$ or $80 \mathrm{~nm}$ long areas of pristine material. Large spacing arrays show "Giant" Shapiro steps in the hundreds-GHz band at $66 \mathrm{~K}$ and are tested as Josephson mixers with improved impedance matching. On the contrary, small spacing arrays behave as one junction with a lower superconducting transition temperature, hence forming a single weak link on distances up to $880 \mathrm{~nm}$. Such design opens a new way to increase the $\mathrm{I}_{c} \mathrm{R}_{n}$ product of the devices, and therefore the efficiency of the Josephson mixers. Hints on the origin of the observed long range proximity effect are proposed.
\end{abstract}

\section{Introduction}

While High Temperature Superconductors (HTSc) have been identified as promising materials to make high frequency $(\mathrm{THz})$ Josephson devices for a long time, recent spectacular developments in the field are making this happening for real [1-3]. The era of THz devices based on HTSc Josephson Junctions (JJ) is opening, and one can explore the potentialities of different techniques able to produce them.

Ion-irradiation technique is a promising approach for manufacturing HTSc integrated circuits [4, 5]. It allows designing a large number of planar JJ arbitrary located on a single superconducting film, and therefore offers a natural scalability compared to other types of HTSc junctions. This technique was first introduced by Tinchev [6] to make RF-SQUIDs. Since then, many other applications were suggested : DC SQUIDs [7], Superconducting Interference Filters (SQIFs) [8, 9], Josephson mixers $[10,11]$. Further developments of this technology have been explored, with other materials such as $\mathrm{MgB}_{2}$ [12], and more recently by using an He Focused Ion Beam to directly creates disorder at a nanoscale to make $\mathrm{JJ}[13,14]$.

The main drawback of the technology for high frequency mixing is the fairly low impedance of the JJ. The normal resistance $R_{n}$ of an ion-irradiated junction is typically of order of a few ohms for standard geometries $(\sim \mu \mathrm{m})$, which does not match the characteristic impedance $Z_{0}(=50 \Omega$ of the readout microwave setup. In Josephson mixers, this has been identified as the main reason for moderate conversion efficiency $[10,11]$. This is also an issue for SQUIDs and SQIFs applications, to obtain a large voltage signal.

One possible way to overcome the problem consists in replacing a single JJ by an array of Josephson junctions in combination with planar coupling structures. Such Josephson Junction Arrays (JJAs) have in addition several advantages that have been described theoretically in the eighties ([15] and references therein), such as a reduced line-width [16] and an enhanced emitted power for instance. Operating prototypes of HTSc array-based devices for different applications have been developed: voltage standard [17, 18], wave form generators [19], detectors and mixers [20,21], for which long range synchronisation of the $\mathrm{JJ}$ has been studied [18, 22-25].

Ion-irradiation technology is capable of delivering reliable arrays on a large scale [8,9,26-28], with up to $36000 \mathrm{JJ}$ in SQUIDs and SQIFs devices [28], and suitable to obtain giant Shapiro steps $[26,29]$ when put in series. A unique property of the irradiation technology lies in the possibility to fabricate very densely packed JJA while keeping a planar geometry. Unlike other types of HTSc arrays, the distance between two adjacent JJ can be on the order of the relevant physical scales such as

*jerome.lesueur@espci.fr 
the proximity effect coherence length $\xi_{N}$, typically a hundred nanometers $[26,29,30]$. It is therefore possible to explore different coupling regimes between close packed JJ, and to address the question of the synchronisation mechanism in these situations [31].

In this article, we report on experimental results on small ion-irradiated JJAs, where the distance between single JJs has been varied from 80 to $960 \mathrm{~nm}$. We clearly identify two regimes : in closely spaced JJ devices, the whole array behaves as a single JJ through a giant proximity effect, while in distant JJ ones, large Shapiro steps are observed as expected for independent JJ in series. Mixing properties of these devices have been studied, together with the physics of closely spaced JJ.

\section{Experiments}

The test-bed geometry that we use to explore the properties of ion-irradiated JJA is a Josephson mixer. As in our previous studies $[10,11]$, the active element (JJ or here a JJA) is embedded in a spiral log-periodic antenna which is inserted in a $50 \Omega$ coplanar waveguide $(\mathrm{CPW})$ transmission line (Figure 1(a)). Heterodyne detection is performed by sending the signal and a Local Oscillator (LO) reference signal onto the antenna (typically in the tens or hundreds $\mathrm{GHz}$ range), and by detecting the intermodulation signal at the Intermediate Frequency (IF), in the $\mathrm{GHz}$ range through the CPW line. Impedance matching with the $50 \Omega$ line and the antenna is a key condition to optimise the conversion efficiency from the high frequency signal to the IF one.
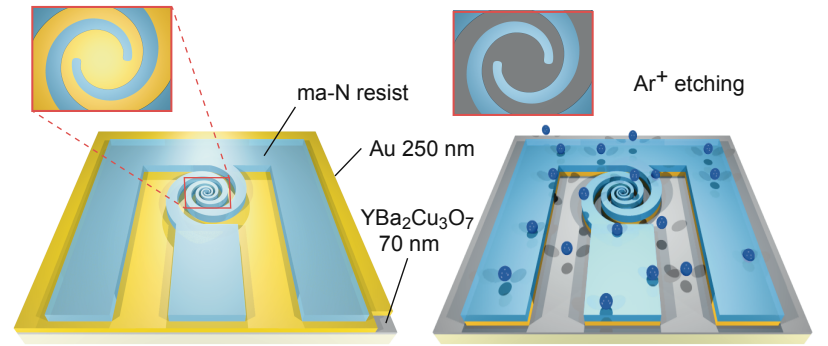

(a)

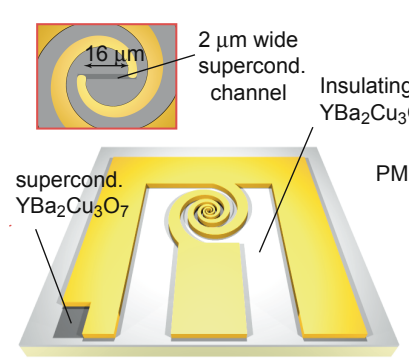

(e) (b)
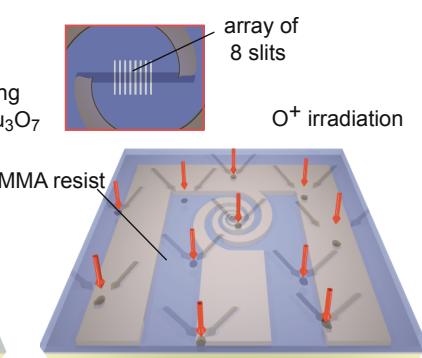

(f)

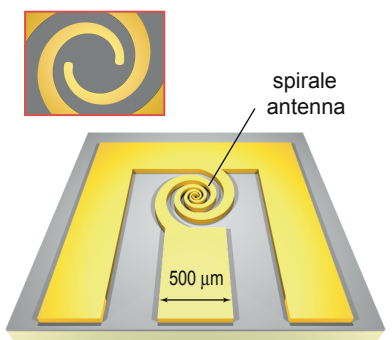

(c)

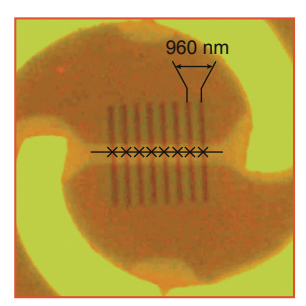

(g)

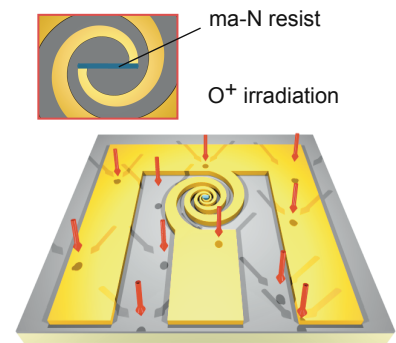

(d)

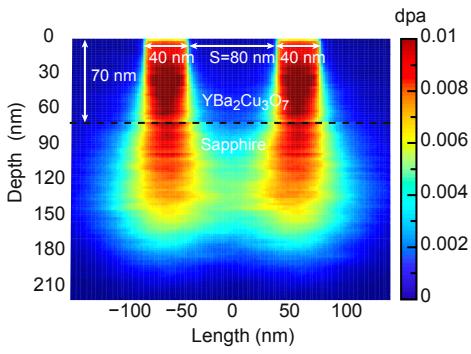

(h)

Figure 1: Illustration of the fabrication process steps : (a) spiral antenna and CPW transmission line patterned in a $500 \mathrm{~nm}$ thick ma-N negative e-beam resist. The sample consists of a 70-nm thick $\mathrm{YBa}_{2} \mathrm{Cu}_{3} \mathrm{O}_{7}$ film grown on sapphire covered by an in situ 250-nm gold layer; (b) 500-eV Ar ion-beam-etching of the gold layer ; (c) gold antenna in the CPW transmission line on $\mathrm{YBa}_{2} \mathrm{Cu}_{3} \mathrm{O}_{7} ;(d)$ high-dose $70-\mathrm{keV}$ oxygen ion irradiation to create insulating regions in exposed $\mathrm{YBa}_{2} \mathrm{Cu}_{3} \mathrm{O}_{7}$. A 2- $\mu \mathrm{m}$ wide channel in the centre of the antenna is protected by a $500 \mathrm{~nm}$ thick ma-N resist mask ; (e) patterned superconducting and insulating $\mathrm{YBa}_{2} \mathrm{Cu}_{3} \mathrm{O}_{7}$ regions ; (f) low-dose $110 \mathrm{keV}$ oxygen ion irradiation of the eight Josephson junctions array patterned as 40-nm-wide slits in a $500 \mathrm{~nm}$ thick PMMA resist ; $(\mathrm{g})$ optical picture of an array of eight $40 \mathrm{~nm}$ wide slits separated by $960 \mathrm{~nm}$, opened in the PMMA resist. The apparent size of the slits is limited by diffraction. Black crosses shows the 8 $\mathrm{JJ}$ in series ; ( $h$ ) SRIM simulation of the defect density (dpa, colour scale) in a $70 \mathrm{~nm}$ thick $\mathrm{YBa}_{2} \mathrm{Cu}_{3} \mathrm{O}_{7}$ film deposited on sapphire substrate, irradiated with $3 \cdot 10^{13}$ at./ $/ \mathrm{cm}^{2}$ through $40 \mathrm{~nm}$ wide slits separated by a spacing $\mathrm{S}=80 \mathrm{~nm}$. 
Details of our standard fabrication technique can be found in Bergeal et al[32] and Malnou et $a l[11]$. The main steps are illustrated in Figure $1(a-f)$, and an optical picture of a N=8 JJA with a spacing $\mathrm{S}=80 \mathrm{~nm}$ is presented in Figure $1(\mathrm{~g})$. In short, we start from a commercial $70 \mathrm{~nm}$ thick $\mathrm{YBa}_{2} \mathrm{Cu}_{3} \mathrm{O}_{7}$ film grown on a sapphire substrate ${ }^{2}$, and covered with a $250 \mathrm{~nm}$ gold layer. The spiral antenna and the CPW transmission line are first defined in the gold layer through a ma-N e-beam patterned resist ${ }^{3}$, followed by a 500-eV $\mathrm{Ar}^{+}$Ion Beam Etching. Then a $2 \mu \mathrm{m}$ wide channel located at the centre of the antenna is patterned in a ma- $\mathrm{N}$ e-beam resist, followed by a $70 \mathrm{keV}$ oxygen ion irradiation at a dose of $1 \cdot 10^{15}$ at $/ \mathrm{cm}^{2}$. This process ensures that the regions of the film which are not protected either by the resist or by the gold layer become insulating. Finally, the junction array is defined by irradiating the superconducting channel through $40-\mathrm{nm}$ wide slits patterned in a PMMA (Poly(methyl methacrylate)) resist, with $110 \mathrm{keV}$ oxygen ions at a dose of $3 \cdot 10^{13}$ at. $/ \mathrm{cm}^{2}$.

SRIM (Stopping and Range of Ions in Matter ) simulations ${ }^{4}$ have been used to evaluate the defect density generated by the ion irradiation. A typical example is displayed in Figure $1(h)$, where the irradiation is performed through two $40 \mathrm{~nm}$ wide slits located $80 \mathrm{~nm}$ apart. Defect density expressed in dpa (displacement per atom, colour scale) is shown within the sample. While it reaches dpa 0.01 in the centre of the slits, and therefore locally decreases $T_{c}$ to form the $\mathrm{JJ}$, it is almost null in the intermediate region. We designed JJA in this limit case, with 4 and $8 \mathrm{JJ}$ in series, and JJA with 960 $\mathrm{nm}$ spacing, with the same number of JJ. Thirteen samples have been measured, and the results that we present here are typical of the whole series.

The samples were placed inside a closed cycle refrigerator, at the focal point of a Winston cone, and then exposed to the external signals through an optical window. CPW lines were connected to microwave readout lines through a PCB (Printed-Circuit Board). The signals in the 4-8 GHz bandwidth were first amplified at cryogenic temperature by a HEMT (High-Electron-Mobility Transistor) low noise amplifier, before further amplification at room temperature. An isolator was placed between the sample and the first amplifier to minimise the back action noise [11]. DC output voltage and AC output power at intermediate frequency $\mathrm{P}_{I F}$ were measured as a function of the applied bias current and temperature.

\section{Arrays with Josephson junctions $960 \mathrm{~nm}$ apart}

First, we focus on the situation where the distance between the slits is much larger $(\mathrm{S}=960 \mathrm{~nm})$ than the typical $80 \mathrm{~nm}$ straggling of the ions calculated by SRIM (see Figure $1(h)$ ), so that the damaged areas do not overlap. In that case junctions can thus be considered as independent. In Figure 2 we show the Resistance $\mathrm{R}$ and the Josephson critical current $I_{c}$ as a function of temperature for arrays with 4 and $8 \mathrm{JJ}$. As expected, $\mathrm{R}(8 \mathrm{JJ}) \sim 2 \times \mathrm{R}(4 \mathrm{JJ})$ and the critical currents look very similar. The coupling temperature $\mathrm{T}_{J} \sim 70 \mathrm{~K}$ below which Josephson coupling takes place is the same for both samples, and corresponds to the one of a single $\mathrm{JJ}$ irradiated in the same conditions. For a given spacing (here $S=960 \mathrm{~nm}$ ), the spread in $\mathrm{T}_{J}$ among the samples is 1 to $2 \mathrm{~K}$ maximum. The spread in resistance and critical current among the samples for a given geometry is $\sim 10 \%$. This points towards rather homogeneous JJ characteristics within the arrays. The I-V characteristics is RSJ (Resistively Shunted Junction) like (see Inset Figure 2) as known for single ion-irradiated JJ [5, 9, 11,32].

The homogeneity is confirmed by the observation of "Giant Shapiro steps" under microwave irradiation. Figure $3(a)$ shows the I-V characteristics of a $4 \mathrm{JJ}$ array under irradiation at $f=20 \mathrm{GHz}$. Shapiro steps correspond to $4 \mathrm{JJ}$ in series $(4 \times f=80 \mathrm{GHz})$. If the spread in $\mathrm{JJ}$ characteristics was two high, we would observed steps at different voltages corresponding to individual junctions. Such Giant Shapiro steps have been already observed in other HTSc JJ $[18,25]$. Extension to arrays with more $\mathrm{JJ}$, up to a thousand, is currently under investigation.

\footnotetext{
${ }^{2}$ Ceraco gmbh.

${ }^{3}$ Micro Resist Technology GmbH

${ }^{4}$ www.srim.org
} 


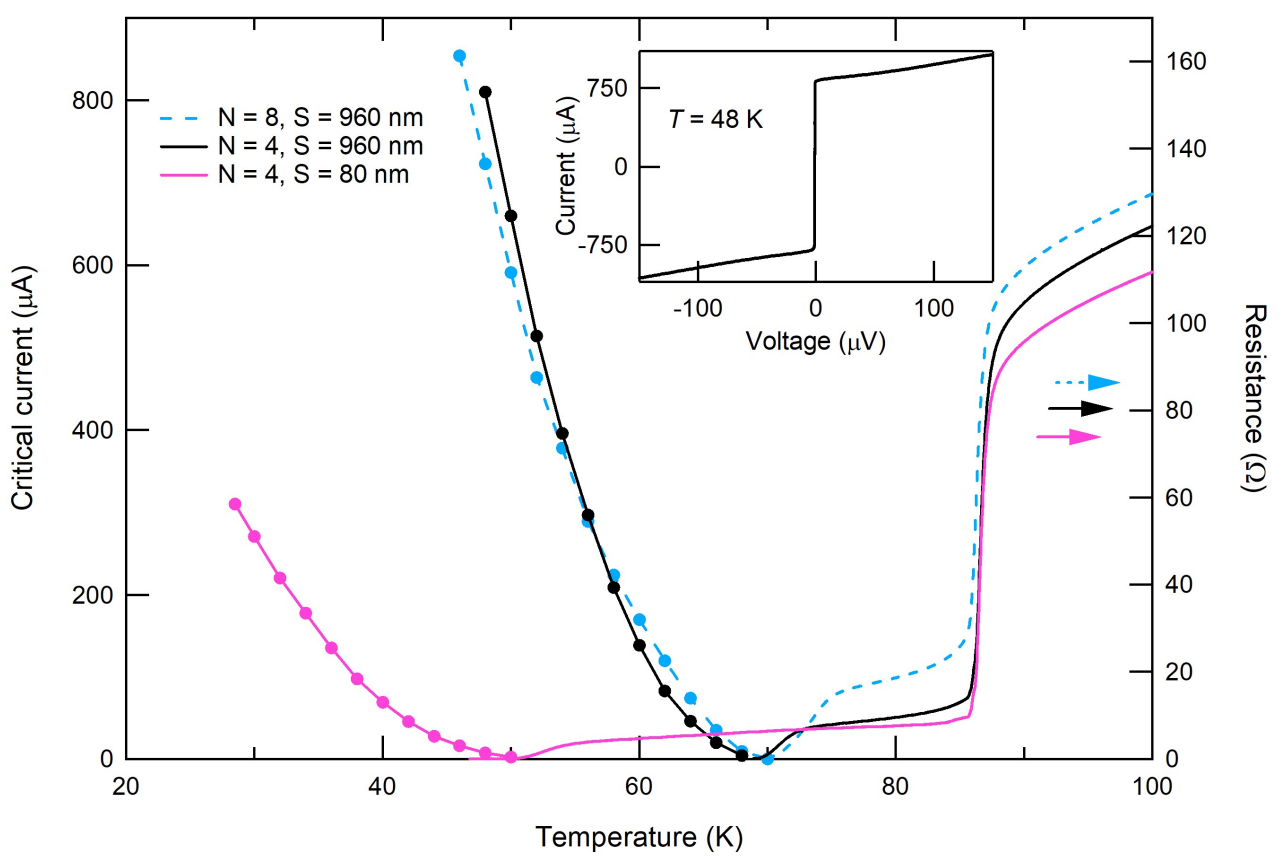

Figure 2: Temperature dependence of the resistance (lines, right scale) and the critical current (symbols, left scale) of JJAs with $\mathrm{N} \mathrm{JJ}$ in series, separated by a spacing $\mathrm{S}$. The transition temperatures $T_{J}$ are $\sim 70 \mathrm{~K}$ and $\sim 50 \mathrm{~K}$ or $\mathrm{S}=960$ and $80 \mathrm{~nm}$, respectively. Inset : I-V characteristics of a $4 \mathrm{JJ}$ array with $\mathrm{S}=960 \mathrm{~nm}$ spacing measured at $48 \mathrm{~K}$, which is RSJ like.

We performed Josephson mixing experiments with these devices. A weak signal at frequency $f_{s}$ was applied to a $4 \mathrm{JJ}$ array $\left(\mathrm{S}=960 \mathrm{~nm}\right.$ ) along with a local oscillator signal at $f_{L O}$, and the available power at intermediate frequency $\mathrm{P}_{I F}$ was measured with a spectrum analyser as a function of an applied DC bias. The result is shown in Figure $4(a)$ for $f_{L O}=20 \mathrm{GHz}$ and $f_{L O}=150 \mathrm{GHz}$. In both cases $f_{s}=f_{L O}-6 \mathrm{GHz}$. The $\mathrm{DC}$ bias $\mathrm{V}$ has been converted into a frequency through the Josephson equation ( $f=V / \phi_{0}$, where $\phi_{0}=h / 2 e$ is the flux quantum), and normalised by $f_{L O}$ and the number $\mathrm{N}$ of $\mathrm{JJ}$ in the array. We observed that $\mathrm{P}_{I F}$ is maximum in the centre of the Shapiro step (only the first one is presented here) for $f_{L O}=20 \mathrm{GHz}$ (black curve), and maximum at the edges of the Shapiro steps for $f_{L O}=150 \mathrm{GHz}$ (orange curve). Such a behaviour has been already reported and analysed for single $\mathrm{JJ}$ in previous publications $[10,11]$, and depends on the characteristic frequency $f_{c}=I_{c} \cdot R_{n} / \phi_{0}$ which rules the dynamics of a JJ, where $R_{n}$ is the normal state resistance. Since $R_{n}$ varies with temperature ( $c f$ Figure 2), we used a special procedure to measure it in the Josephson regime below $T_{J}$, detailed and justified by Malnou et al[10,11]. $R_{n}(\mathrm{~T})$ is the normal state resistance measured under RF power strong enough to fully suppress the critical current $I_{c}$ at temperature T. This value coincides with the extrapolation to $T<T_{J}$ of the resistance curve measured above $T_{J}$. For the sample reported in Figure 4, $f_{c} \sim 100 \mathrm{GHz}$ which is between $20 \mathrm{GHz}$ (black curve) and 150 $\mathrm{GHz}$ (orange curve). This shows that JJA mixers with spaced JJ behave like single JJ ones.

This is true for temperature just below $\mathrm{T}_{J}(\mathrm{~T}=66 \mathrm{~K}$ for the data in Figure $3(a)$ and Figure 4 (a)), but a more chaotic behaviour is observed when the temperature is lowered. This is shown by observing the mixing patterns at "high" $66 \mathrm{~K}$ and "low" $60 \mathrm{~K}$ temperatures displayed in Figure4 (b) and $(c)$ respectively. The $960 \mathrm{~nm}$ JJA with $4 \mathrm{JJ}$ has been irradiated with $f_{s}=14 \mathrm{GHz}$ and $f_{L O}=$ $20 \mathrm{GHz}$ microwaves. The output power at the intermediate frequency $\mathrm{P}_{I F}$ is represented in colour scale as a function of both the DC bias voltage (converted into frequency $f$ as before) and the local oscillator power $\mathrm{P}_{L O}$. At high temperature (Figure $4(c)$ ), we observe a regular pattern with oscillations separated by $80 \mathrm{GHz}(=4 \times 20 \mathrm{GHz})$, as well as a non-monotonic modulation with $\mathrm{P}_{L O}$, as expected. At lower temperature (Fig. 4(b)), the pattern becomes more fuzzy, with oscillations corresponding 
(a)

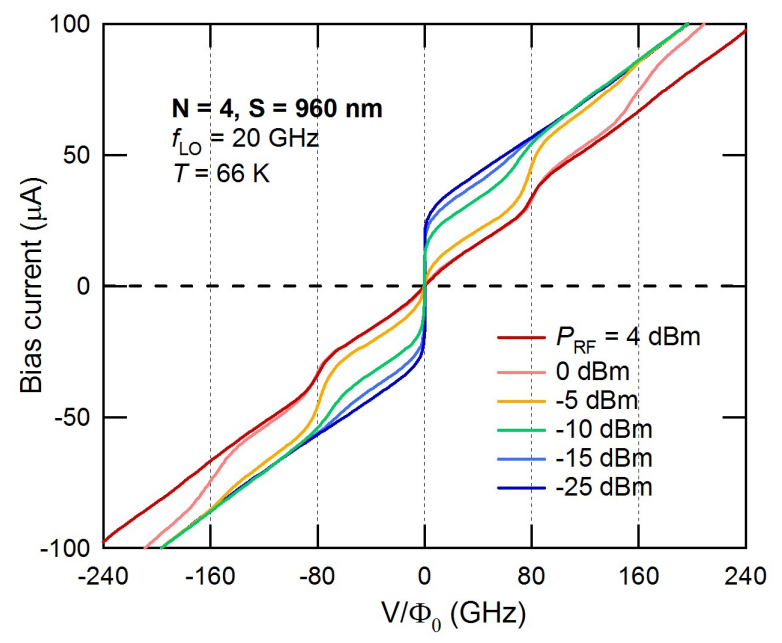

(b)

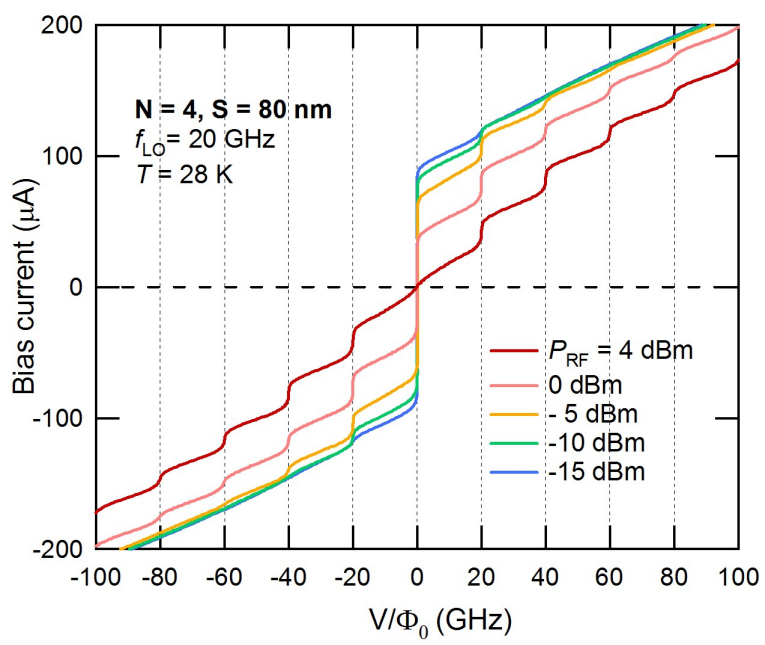

Figure 3: DC current versus voltage converted into Josephson frequency - expressed in Volts $/ \Phi_{0}\left(\Phi_{0}\right.$ is the flux quantum) - of $4 \mathrm{JJ}$ arrays with $\mathrm{S}=960 \mathrm{~nm}((a)$, left panel) and $\mathrm{S}=80 \mathrm{~nm}((b)$, right panel) under microwave irradiation at $f_{L O}=20 \mathrm{GHz}$, for different power $P_{R F}$. "Giant Shapiro steps" appear for $\mathrm{S}=960 \mathrm{~nm}$. In both cases, I-V characteristics are like.

to individual $\mathrm{JJ}$ or group of JJ. This is because the scattering of $\mathrm{JJ}$ parameters is too high. Indeed, sufficient uniformity between junctions is necessary to provide equal optimal biasing for all junctions in the array, and to obtain Giant Shapiro steps. Scattering is higher at low temperature because of the rather steep dependence of the critical current $I_{c}$ with temperature (Figure 2). A tiny difference between the critical currents close to $T_{J}$ translates into an increasing difference as the temperature is lowered.

The first result of this study is that, in a certain range of temperature, one can benefit from the larger impedance of series-JJA to better match the impedance of the circuits, while preserving good mixing properties in these devices. This does not require phase locking between the JJ [33]. However, mutual phase locking would allow to decrease the line-width of the junctions self-oscillation [16], and therefore decrease the noise temperature of the Josephson mixer. Indeed, the local oscillator generates additional noise in the output IF band by down conversion of wide Josephson self-oscillations (see e.g. [15]). The fact that short range interaction can lead in some cases to mutual phase locking [34-37], and that parameters scattering in our arrays allows to obtain Giant Shapiro steps encouraged us to put slits as close as possible to keep damaged areas still separated but with shorter virgin areas.

\section{Arrays with Josephson junctions $80 \mathrm{~nm}$ apart}

Samples with 4 and $8 \mathrm{JJ}$ in series - spaced by $80 \mathrm{~nm}$ - have been also made and studied. The resistance and the critical current of a typical $4 \mathrm{JJ}$ device are plotted as a function of temperature in Figure 2. Surprisingly, the Josephson coupling temperature is significantly lower $\left(\mathrm{T}_{J} \sim 50 \mathrm{~K}\right)$ than for $960 \mathrm{~nm}$ spaced JJA. The resistance drops to zero in a single step as for $960 \mathrm{~nm}$ spaced array. That means that all the $\mathrm{JJ}$ have the same $\mathrm{T}_{J}$, and that the behaviour of the array is not dominated by a single peculiar JJ. In the opposite case, we should see multiple steps in the $\mathrm{R}(\mathrm{T})$ curves since it is a series array. The resistance above $\mathrm{T}_{J}$ is comparable in both types of samples, while $I_{c}(\mathrm{~T})$ shows a less steep increase when the temperature is lowered. When irradiated with $f=20 \mathrm{GHz}$ microwaves, regular Shapiro steps displaying a modulation with RF power as expected, develop in the I-V characteristics, whose length correspond to $f$, and not $4 \times f$ as previously observed in the $960 \mathrm{~nm}$ JJA (Figure $3(b)$ ). 
The same behaviour is observed for $\mathrm{N}=8 \mathrm{JJA}$ (not shown). This takes place in the whole Josephson temperature range (from $28 \mathrm{~K}$ to $50 \mathrm{~K}$ ). Therefore, JJAs behave as single $\mathrm{JJ}$ with a lower $\mathrm{T}_{J}$, and a resistance given by the sum of the 4 or $8 \mathrm{JJ}$ in series. This indicates that Josephson coupling is established across the whole array, that is over a very large distance : $400 \mathrm{~nm}$ in the $4 \mathrm{JJ}$ case, and $880 \mathrm{~nm}$ in the $8 \mathrm{JJ}$ one. While such a long distance Josephson coupling is by itself an interesting experimental fact that deserves a special discussion (see below), let us emphasize another important outcome of this result for high frequency mixing.

In a mixer, one defines the conversion efficiency $\eta$ as the ratio between the power at the intermediate frequency $P_{I F}$ and the incident signal power $P_{s}\left(\eta=P_{I F} / P_{s}\right)$. It gives the ability of the mixer to efficiently down-convert high frequency signals into low frequency ones. For standard Josephson mixers [38], $\eta$ is proportional to $\left(f_{c} / f_{L O}\right)^{2}$, where $f_{c}=I_{c} \cdot R_{n} / \Phi_{0}$ is the characteristic frequency of the $\mathrm{JJ}$ as already defined. Malnou et al. recently showed that this applies to $\mathrm{YBa}_{2} \mathrm{Cu}_{3} \mathrm{O}_{7}$ ion irradiated JJ $[10,11]$. Since $\eta$ increases quadratically with $R_{n}$, the discovery of series JJAs with up to 8 $\mathrm{JJ}$ behaving as a single one opens new perspectives to make high efficiency Josephson mixers with HTSc, even if the critical current is lowered.
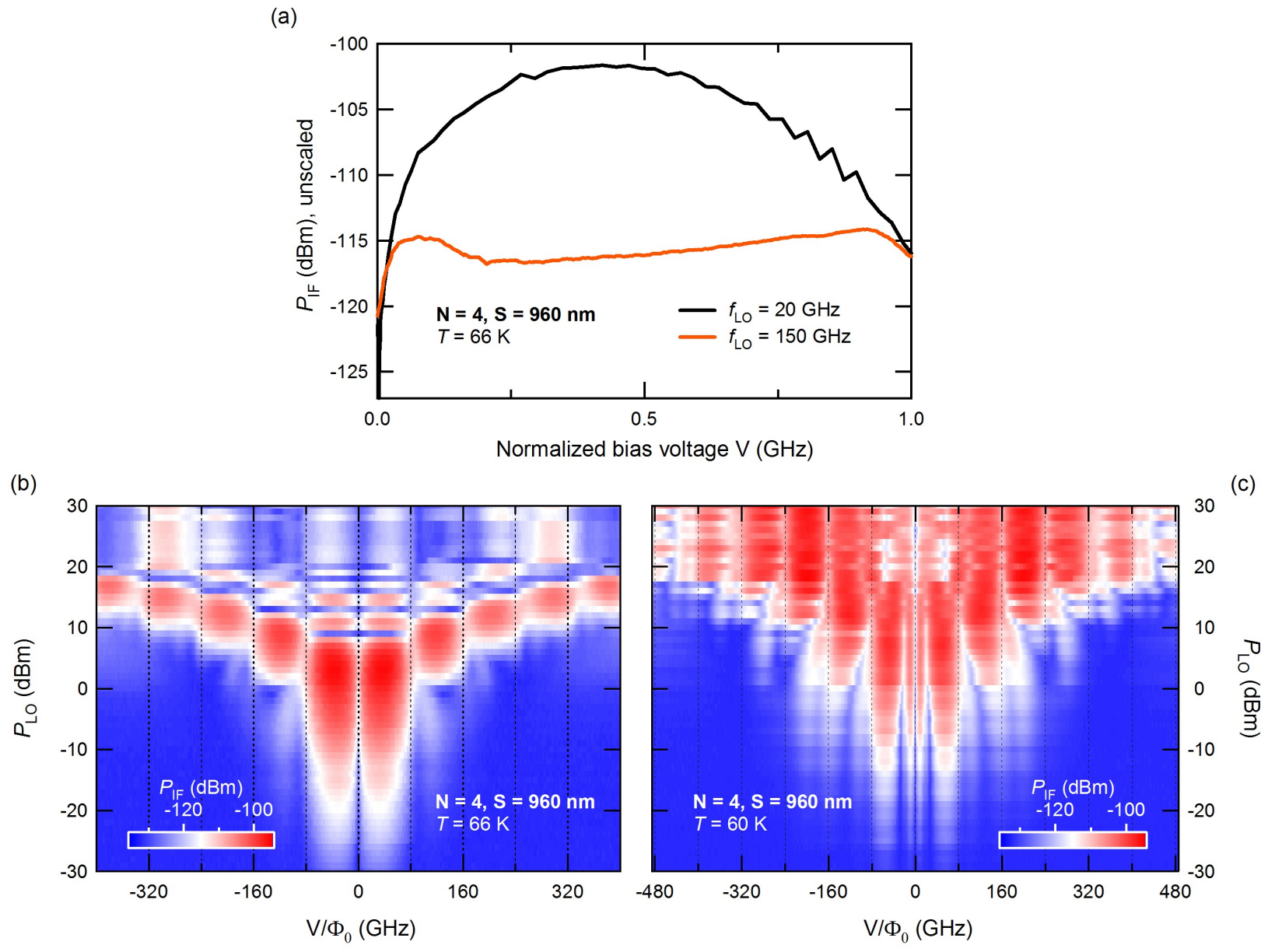

Figure 4: (a) Power at the intermediate frequency $P_{I F}=6 \mathrm{GHz}$ as a function of the bias voltage converted into frequency and normalised by the Local Oscillator frequency $f_{L O}$ and the number of $\mathrm{JJ}$ (see text), for a $960 \mathrm{~nm}$ spaced $4 \mathrm{JJ}$ array, measured at $\mathrm{T}=66 \mathrm{~K}$. In black, $f_{L O}=20 \mathrm{GHz}$ and signal frequency $f_{s}=14 \mathrm{GHz}$. In orange, $f_{L O}=150 \mathrm{GHz}$ and $f_{s}=144 \mathrm{GHz}$. (b) $P_{I F}=6 \mathrm{GHz}$ on a colour scale as a function of both the bias voltage converted into frequency (see text) and the Local Oscillator power $P_{L O}$ for the same JJA at $\mathrm{T}=66 \mathrm{~K}$. Periodicity in frequency is $80 \mathrm{GHz}$, that is $4 \times 20 G H z$ : these are "Giant Shapiro steps". (c) Same data measured at $\mathrm{T}=$ $60 \mathrm{~K}$. The pattern is more fuzzy. 


\section{Discussion}

As reported above, closely spaced ( $80 \mathrm{~nm}$ ) JJA behave as a single JJ where the coupling between superconducting reservoirs takes place over very long distances, up to $880 \mathrm{~nm}$. This behaviour has been observed on the different samples that we measured. We carefully checked that no short-cut could explain such a behavior by direct observation of optical pictures. DC and RF measurements, including mixing, show that the whole array behaves as a giant JJ. Indeed, the observed Shapiro steps and the high frequency mixing can only occur if a phase coherent behaviour takes place across the array. That means that some kind of long range proximity effect occurs in HTSc, which is quite surprising in these materials. Proximity effect, firstly introduced by de Gennes [39], describes the coherent propagation of Cooper pairs within non-superconducting regions called "Normal" over a distance $\xi_{N}$, which eventually insures the Josephson coupling through this barrier (SNS junctions) at a temperature $\mathrm{T}_{J}$. The latter decreases as a function of the ratio $l / \xi_{N}$, where $l$ is the length of the Normal region. Ion-irradiated JJ have been shown to belong to this category $[4,5,40,41]$. Although this theory was not originally developed for d-wave High- $\mathrm{T}_{c}$ Superconductors, it has been successfully used to describe different properties of irradiated $\mathrm{YBa}_{2} \mathrm{Cu}_{3} \mathrm{O}_{7} \mathrm{JJ}[5,16,41-43]$. In case the "Normal" part is a superconductor with a lower critical temperature $\mathrm{T}_{c}^{\prime}$, as for the damaged region in ion-irradiated JJ, $\xi_{N}$ goes as $\sqrt{1+\frac{2}{\ln \frac{T}{T_{c}^{\prime}}}}$, and therefore diverges at $\mathrm{T}_{c}^{\prime}[39]$.

Previous experiments on $5 \mu \mathrm{m}$ wide bridges irradiated with the same dose $\left(3 \cdot 10^{13} \mathrm{at} . / \mathrm{cm}^{2}\right)$ through slits of different width $w$ showed that the Josephson temperature $\mathrm{T}_{J}$ strongly depends on the width of the slit until it reaches a saturation value [44]. This can be seen in Figure 5 where both the resistance $\mathrm{R}$ and the critical current $\mathrm{I}_{c}$ have been plotted as a function of temperature for increasing $w$, from 20 to $1000 \mathrm{~nm}$. For $w<200 \mathrm{~nm}$, they change significantly while they saturate for $w>500 \mathrm{~nm}$. That means that for a critical value $w_{c}$, which is between 200 and $500 \mathrm{~nm}, \mathrm{~T}_{J}$ reaches $\mathrm{T}_{c}^{\prime}$. This sets an upper bound for $\xi_{N}$ is these conditions, which should be around 100-200 $\mathrm{nm}$.

We can thus understand different features of our present data. Clearly, $960 \mathrm{~nm}$ is much larger than $\xi_{N}$, and $960 \mathrm{~nm}$ spaced $\mathrm{JJ}$ are completely independent, while $80 \mathrm{~nm}$ ones can have some interaction. In fact, as proximity effect relates to the propagation of superconducting correlations over $\xi_{N}$, inverse proximity effect relates to normal quasiparticles diffusing in the superconducting part, on the same extension, lowering superconductivity in this region. Therefore, when the distance between two diffusive SNS JJ is smaller than $\xi_{N}$, they appear as a single JJ with an apparent increased length. This can be seen in Figure 5 where $\mathrm{I}_{c}(\mathrm{~T})$ and $\mathrm{R}(\mathrm{T})$ for the $80 \mathrm{~nm}$ spaced JJA (total length $400 \mathrm{~nm}$ for $4 \mathrm{JJ}$ in that case) are close to the one of $500 \mathrm{~nm}$ wide $\mathrm{JJ}$ of the previous experiment, with almost the same $\mathrm{T}_{J}$. Note that $\mathrm{T}_{J}$ for the $8 \mathrm{JJA}$ of total length $880 \mathrm{~nm}$ is the same because of the saturation effect mentioned above, and that $\mathrm{T}_{J}$ for $960 \mathrm{~nm}$ spaced JJA is close to the one of single $20 \mathrm{~nm}$ wide one.

In the literature, only two series of experiments have been reported with closely spaced $\mathrm{YBa}_{2} \mathrm{Cu}_{3} \mathrm{O}_{7}$ planar JJ. Using direct e-beam irradiation to fabricate JJ, Booij et al [30] studied the electro-dynamics of two JJ separated by $75 \mathrm{~nm}$ when an external magnetic field is applied. They essentially discuss the current redistribution within the electrodes that form the JJ, and its consequences on the Fraunhofer pattern. However, there are no measurement at finite frequency that we can compare with. Chen et al used an ion-irradiation technique very close to ours, to make pairs and arrays of $\mathrm{JJ}$ with spacing ranging from $125 \mathrm{~nm}$ to $800 \mathrm{~nm}[26,45]$, and studied their $\mathrm{AC}$ response. They observed Giant Shapiro steps in structures with inter-JJ distances larger than $150 \mathrm{~nm}$. Our results on $960 \mathrm{~nm}$ spaced JJ are fully compatible with theirs. When the distance between JJ is smaller than $150 \mathrm{~nm}$, Chen et al report on a different and very interesting behaviour[45]. At low RF power, samples display Giant Shapiro steps corresponding to independent JJ. However at high RF power, it behaves like a single JJ, exactly as our $80 \mathrm{~nm}$ spaced JJA. This behaviour can be understood in the frame that we proposed above. The distance between JJ in the array of Chen et alis $150 \mathrm{~nm}$, that is on the order of $\xi_{N}$, may be a little bit larger : the JJ are thus decoupled. But at the same time, the inverse proximity 
effect decreases the strength of superconductivity in the region between the JJ. Increasing RF power introduces incoherent processes (analogous to temperature), and this region becomes a weak link as well. The whole structure behaves as a single JJ.

Indeed, these are qualitative arguments only, and one needs to understand in more detail the mechanism underlying this surprising result. A microscopic theory based on semi-classical Usadel equations can be developed[44]. Recently a model has been proposed by Kresin et al [46,47], to explain a Giant proximity effect reported in c-axis transport measurement in cuprates [48]. The main idea is that two distanced superconductors separated by a chain of superconducting islands with their own pairing functions might form a single Josephson junction if their pairing amplitudes overlap. Application of this theory to the present data needs to be done.
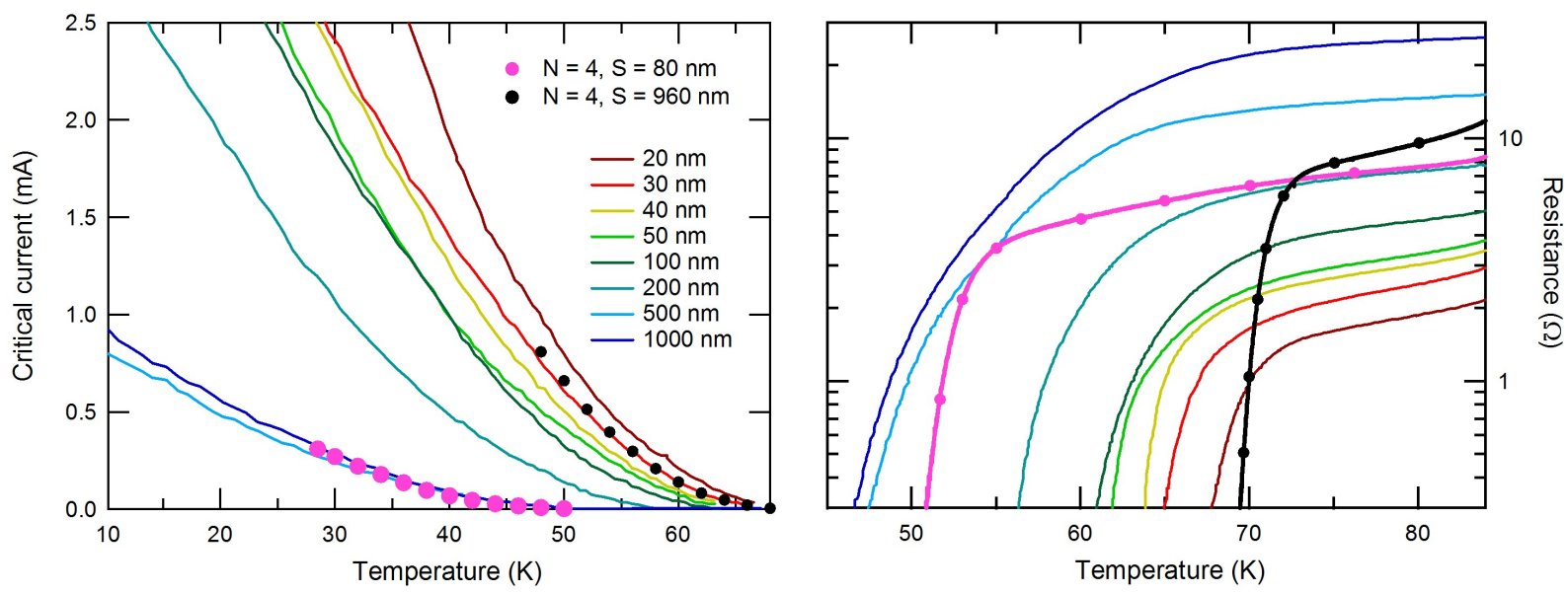

Figure 5: Temperature dependence of the critical current $\mathrm{I}_{c}$ (left panel) and the resistance $\mathrm{R}$ (right panel) of $\mathrm{YBa}_{2} \mathrm{Cu}_{3} \mathrm{O}_{7} 5 \mu \mathrm{m}$ wide bridges irradiated through slits of different width $w$ with the same dose $\left(3 \cdot 10^{13}\right.$ at. $\left./ \mathrm{cm}^{2}\right)$. $w$ ranges from 20 to $1000 \mathrm{~nm}$ (see colour code in the panel). For comparison, $\mathrm{I}_{c}(\mathrm{~T})$ and $\mathrm{R}(\mathrm{T})$ for the 4JJ arrays are also shown, for $\mathrm{S}=80 \mathrm{~nm}$ (pink solid circle and line) and $960 \mathrm{~nm}$ (black solid circle and line).

\section{Conclusion}

Josephson mixing on Giant Shapiro steps of ion-irradiated Josephson junctions arrays has been demonstrated. Replacing a single junction by an array will allow us to improve impedance mismatch of previously reported Josephson mixers $[10,11]$. To improve noise temperature of the mixer as well as for many other possible applications, phase locking between junctions in an array is necessary. We believe that for this particular type of junctions, short-range interaction may be a promising approach to achieve phase synchronisation.

The reported experiments showed that $80 \mathrm{~nm}$-spaced JJA behave as single junction. From an application point of view, this offers an interesting possibility to increase the $\mathrm{I}_{c} \mathrm{R}_{N}$ product of ionirradiated JJs, and correlatively the conversion efficiency of the Josephson mixers. On the basic science side, long range proximity effect over distances up to $880 \mathrm{~nm}$ observed here can be explained by general arguments taking into account the inverse proximity effect and the estimated value of the Normal coherence length. 


\section{Acknowledgments}

The authors thank Yann Legall for ion irradiations. Useful comments of V. Kresin, Yu. Ovchinnickov and V. Kornev are greatly appreciated. This work has been supported by the T-SUN ANR ASTRID program (ANR-13-ASTR-0025-01), the Emergence program Contract of Ville de Paris and by the Région Ile-de-France in the framework of the DIM Nano-K and Sesame programs.

\section{References}

[1] J. Du, A. R. Weily, X. Gao, T. Zhang, C. P. Foley, and Y. J. Guo, "HTS step-edge Josephson junction terahertz harmonic mixer," Superconductor Science and Technology, vol. 30, no. 2, p. 024002, 2016.

[2] A. V. Snezhko, I. I. Gundareva, M. V. Lyatti, O. Y. Volkov, V. V. Pavlovskiy, U. Poppe, and Y. Y. Divin, "Terahertz Josephson spectral analysis and its applications," Superconductor Science and Technology, vol. 30, no. 4, p. 044001, 2017.

[3] J. Du, C. M. Pegrum, X. Gao, A. R. Weily, T. Zhang, Y. J. Guo, and C. P. Foley, "Harmonic Mixing Using a HTS Step-Edge Josephson Junction at 0.6 THz Frequency," IEEE Transactions on Applied Superconductivity, vol. 27, p. 1500905, 2017.

[4] A. S. Katz, A. Sun, S. I. Woods, and R. C. Dynes, "Planar thin film $\mathrm{YBa}_{2} \mathrm{Cu}_{3} \mathrm{O}_{7-\delta}$ Josephson junctions via nanolithography and ion damage," Applied Physics Letters, vol. 72, no. 16, p. 2032, 1998.

[5] N. Bergeal, X. Grison, J. Lesueur, G. Faini, M. Aprili, and J. Contour, "High-quality planar high-T ${ }_{c}$ Josephson junctions," Applied Physics Letters, vol. 87, p. 102502, 2005.

[6] S. Tinchev, "Investigation of RF SQUIDs made from epitaxial YBCO films," Superconductor Science and Technology, vol. 3, no. 10, p. 500, 1990.

[7] N. Bergeal, J. Lesueur, G. Faini, M. Aprili, and J. Contour, "High T $_{c}$ superconducting quantum interference devices made by ion irradiation," Applied Physics Letters, vol. 89, p. 112515, 2006.

[8] S. Cybart, S. Anton, S. Wu, J. Clarke, and R. Dynes, "Very large scale integration of nanopatterned $\mathrm{YBa}_{2} \mathrm{Cu}_{3} \mathrm{O}_{7-\delta}$ Josephson junctions in two-dimensional array," Nano Letters, vol. 9, no. 10, p. 3581, 2009.

[9] S. Ouanani, J. Kermorvant, C. Ulysse, M. Malnou, Y. Lemaître, B. Marcilhac, C. FeuilletPalma, D. Bergeal, N. Crété, and J. Lesueur, "High Tc Superconducting Quantum Interference Filters (SQIFs) made by ion irradiation," Superconductor Science and Technology, vol. 29, no. 9, p. 094002, 2016.

[10] M. Malnou, A. Luo, T. Wolf, Y. Wang, C. Feuillet-Palma, C. Ulysse, G. Faini, P. Febvre, M. Sirena, J. Lesueur, and N. Bergeal, "Toward terahertz heterodyne detection with superconducting josephson junctions," Applied Physics Letters, vol. 101, no. 23, p. 233505, 2012.

[11] M. Malnou, C. Feuillet-Palma, C. Ulysse, G. Faini, P. Febvre, M. Sirena, L. Olanier, J. Lesueur, and N. Bergeal, "High- $\mathrm{T}_{c}$ superconducting josephson mixers for terahertz heterodyne detection," Journal of Applied Physics, vol. 116, p. 074505, 2014.

[12] S. Cybart, K. Chen, Y. Cui, Q. Li, X. Xi, and R. Dynes, "Planar MgB ${ }_{2}$ Josephson junctions and series arrays via nanolithography and ion damage," Applied Physics Letters, vol. 88, p. 012509, 2006. 
[13] S. Cybart, E. Cho, T. Wong, H. Björn, M. Ma, C. Huynh, and R. Dynes, "Nano Josephson superconducting tunnel junctions in $\mathrm{YBa}_{2} \mathrm{Cu}_{3} \mathrm{O}_{7-\delta}$ directly patterned with a focused helium ion beam," Nature Nanotechnology, vol. 10, p. 598, 2015.

[14] E. Cho, M. Ma, C. Huynh, K. Pratt, D. Paulson, V. Glyantsev, R. Dynes, and S. Cybart, " $\mathrm{YBa}_{2} \mathrm{Cu}_{3} \mathrm{O}_{7-\delta}$ superconducting quantum interference devices with metallic to insulating barriers written with a focused helium ion beam," Applied Physics Letters, vol. 106, p. 252601, 2015.

[15] A. Jain, K. Likharev, J. Lukens, and J. Sauvageau, "Mutual phase-locking in Josephson junction arrays," Physics Reports, vol. 109, no. 6, p. 309, 1984.

[16] A. Sharafiev, M. Malnou, C. Feuillet-Palma, C. Ulysse, P. Febvre, J. Lesueur, and N. Bergeal, "Josephson oscillation linewidth of ion-irradiated $\mathrm{YBa}_{2} \mathrm{Cu}_{3} \mathrm{O}_{7}$ junctions," Superconductor Science and Technology, vol. 29, no. 7, p. 074001, 2016.

[17] S. Benz and C. Hamilton, "A pulse-driven programmable Josephson voltage standard," Applied Physics Letters, vol. 68, p. 3171, 1996.

[18] A. Klushin, W. Prusseit, E. Sodtke, S. I. Borovitskii, L. E. Amatuni, and H. Kohlstedt, "Shunted bicrystal Josephson junctions arrays for voltage standards," Applied Physics Letters, vol. 69, p. $1634,1996$.

[19] S. Benz, C. Hamilton, C. Burroughs, T. Harvey, A. Lawrence, and J. Przybysz, "Pulse-driven Josephson Digital/Analog Converter," IEEE Transactions on Applied Superconductivity, vol. 8, no. 2, 1998.

[20] J. Konopka, I. Wolff, S. Beuven, and M. Siege, "Mixing and detection in YBaCuO step-edge Josephson junction arrays up to $670 \mathrm{GHz}$," IEEE Transactions on Applied Superconductivity, vol. 5, no. 2, p. 2443, 1995.

[21] K. Tsuru, K. Miyahara, and M. Suzuki, "Millimeter wave response of Josephson junction arrays using a waveguide to microstrip line converter," in Advances in Superconductivity-VIII (H. Hayakawa and Y. Enomoto, eds.), p. 1171, Springer, 1995.

[22] P. Barbara, A. Cawthorne, S. Shitov, and C. Lobb, "Stimulated emission and amplification in Josephson junctions arrays," Physical Review Letters, vol. 82, no. 9, p. 1963, 1999.

[23] S. Han, B. Bi, W. Zhang, and J. Lukens, "Demonstration of josephson effect submillimeter wave sources with increased power," Applied Physics Letters, vol. 64, p. 1963, 1994.

[24] K. Constantinian, A. Mashtakov, G. Ovsyannikov, V. Kornev, A. Arzumanov, N. Shcherbakov, M. Darula, J. Mygind, and N. Pedersen, "MM wave Josephson radiation in High- $\mathrm{T}_{c}$ bicrystal junction arrays," in Proc. EUCAS 99 conference, IOP Publishing Ltd, 2000.

[25] A. Klushin, M. He, M. Levitchev, and N. Kurin, V.V. Klein, "Optimization of the coupling of

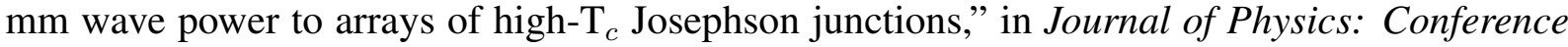
Series, vol. 97, p. 012268, IOP Publishing, 2008.

[26] K. Chen, S. Cybart, and R. Dynes, "Planar thin film $\mathrm{YBa}_{2} \mathrm{Cu}_{3} \mathrm{O}_{7}$ Josephson junction pairs and arrays via nanolithography and ion damage," Applied Physics Letters, vol. 85, no. 14, p. 2863, 2004.

[27] S. Cybart, S. Wu, S. Anton, I. Siddiqi, J. Clarke, and R. Dynes, "Series array of incommensurate superconducting quantum interference devices from $\mathrm{YBa}_{2} \mathrm{Cu}_{3} \mathrm{O}_{7}$ ion damage Josephson junctions," Applied Physics Letters, vol. 93, p. 182502, 2008. 
[28] S. A. Cybart, E. Y. Cho, T. J. Wong, V. N. Glyantsev, J. U. Huh, C. S. Yung, B. H. Moeckly, J. W. Beeman, E. Ulin-Avila, S. M. Wu, and R. C. Dynes, "Large voltage modulation in magnetic field sensors from two-dimensional arrays of Y-Ba-Cu-O nano Josephson junctions," Applied Physics Letters, vol. 104, no. 6, p. 062601, 2014.

[29] S. Cybart, K. Chen, and R. Dynes, "Planar $\mathrm{MgB}_{2}$ Josephson junctions and series arrays via nanolithography and ion damage," IEEE Transactions on Applied Superconductivity, vol. 15, no. 2 , p. $241,2005$.

[30] W. E. Booij, A. J. Pauza, D. F. Moore, E. J. Tarte, and M. G. Blamire, "Electrodynamics of closely coupled $\mathrm{YBa}_{2} \mathrm{Cu}_{3} \mathrm{O}_{7-\delta}$ unctions," IEEE Transactions on Applied Superconductivity, vol. 7, p. 3025, 1997.

[31] J. Bindslev Hansen and P. Lindelof, "Static and dynamic interactions beween Josephson junctions," Review of Modern Physics, vol. 56, no. 3, p. 431, 1984.

[32] N. Bergeal, J. Lesueur, M. Sirena, G. Faini, M. Aprili, J. P. Contour, and B. Leridon, "Using ion irradiation to make high-T ${ }_{c}$ Josephson junctions," Journal of Applied Physics, vol. 102, no. 8, p. 083903, 2007.

[33] L. Kuzmin, K. Likharev, and V. Migulin, "Microwave receivers using SQUIDs and Josephson junction arrays," IEEE Transactions on Magnetics, vol. MAG-17, no. 1, p. 822, 1981.

[34] P. Lindelof, B. Hansen, J. Mygind, N. Pedersen, and O. Sørensen, "Coherent Josephson radiation from an array of two Dayem briges," Physics Letters, vol. 60A, no. 5, 1977.

[35] H. Li, R. Ono, L. Vale, D. Rudman, and S. Liou, "Interactions between bicrystal Josephson junctions in a multilayer structure," IEEE Transactions on Applied Superconductivity, vol. 9, no. $2,1999$.

[36] R. Kleiner, F. Steinmeyer, G. Kunkel, and P. Müller, "Intrinsic Josephson effects in $\mathrm{Bi}_{2} \mathrm{Sr}_{2} \mathrm{CaCu}_{2} \mathrm{O}_{8}$ single crystals," Physical Review Letters, vol. 68, p. 2394, 1992.

[37] G. Carapella, G. Costabile, A. Petraglia, N. Pedersen, and J. Mygind, "Phase locked fluxonantifluxon states in stacked Josephson junctions," Applied Physics Letters, vol. 69, p. 1300, 1996.

[38] A. Barone and G. Paterno, Physics and applications of the Josephson effect. USA: A WileyInterscience publication, 1982.

[39] P. de Gennes, "Boundary effects in superconductors," Review of Modern Physics, vol. 36, p. 225, 1964.

[40] S. Tinchev, "Current-phase relation in high- $\mathrm{T}_{c}$ weak links made by oxygen-ion irradiation," Physica C, p. 173, 1993.

[41] A. S. Katz, S. I. Woods, and R. C. Dynes, "Transport properties of high-T ${ }_{c}$ planar josephson junctions fabricated by nanolithography and ion implantation," Journal of Applied Physics, vol. 87, p. 2978, 2000.

[42] A. Pauza, W. Booij, H. K., D. Moore, M. Blamire, R. A., and V. L.R., "Electron-beam damaged high-temperature superconductor josephson junctions," Journal of Applied Physics, no. 21, p. 5612, 1997. 
[43] W. Booij, A. Pauza, E. Tarte, D. Moore, and M. Blamire, "Proximity coupling in high- $t_{c}$ josephson junctions produced by focused electron beam irradiation," Physical Review B, vol. 55, no. 21 , p. 1634, 1997.

[44] T. Wolf, Étude de nanojunctions Josephson à haute température critique en vue d'applications térahertz. PhD thesis, University Pierre and Marie Curie, 2010. In French.

[45] K. Chen, S. Cybart, and R. Dynes, "Study of closely spaced $\mathrm{YBa}_{2} \mathrm{Cu}_{3} \mathrm{O}_{7-\delta}$ Josephson junction pairs," IEEE Transactions on Applied Superconductivity, vol. 15, no. 2, p. 149, 2005.

[46] V. Kresin, Y. Ovchinnikov, and S. Wolf, "Giant Josephson proximity effect," Applied Physics Letters, vol. 83, no. 4, p. 722, 2003.

[47] V. Kresin, Y. Ovchinnikov, and S. Wolf, "Inhomogeneous superconductivity and the "pseudogap" state of novel superconductors," Physics Reports, vol. 431, p. 231, 2006.

[48] I. Bozovic, G. Logvenov, M. Verhoeven, P. Caputo, E. Goldobin, and M. Beasley, "Giant proximity effect in cuprate superconductors," Physical Review Letters, vol. 93, no. 15, 2004. 